\title{
ON THE ARITHMETIC PROPERTIES OF THE VALUES OF G-FUNCTIONS
}

\author{
K. VÄÄNÄNEN and XU GUANGSHAN
}

(Received 7 September 1987)

Communicated by J. H. Loxton

\begin{abstract}
In a recent paper Chudnovsky considered the arithmetic properties of certain values of classical Siegel $G$-function solutions of a system of linear homogeneous differential equations without any restrictive conditions. The present paper generalizes some results of Chudnovsky in both the archimedian and the $p$-adic case.
\end{abstract}

1980 Mathematics subject classification (Amer. Math. Soc.) (1985 Revision): 10 F 35.

In 1929 Siegel [9] developed a method for studying the arithmetic properties of the values of certain classes of analytic functions, called $E$ - and $G$-functions. Later this method has been applied to $G$-functions by Nurmagomedov [8], Galochkin [5], Flicker [4], Väänänen ([10], [11], [12], [13]), Matveev [6] and $\mathrm{Xu}$ ([15], [16]), for example, but their results use the additional Galochkin's [5] condition. This is replaced by another condition in an important work of Bombieri [2]. Then, in a recent paper, Chudnovsky [3], using ingenious new ideas, succeeded in considering the arithmetic properties of the values of classical $G$-function solutions of a system of linear homogeneous differential equations without any restrictive conditions.

In Väänänen and Xu [14] some generalizations of certain results of Chudnovsky [3] are obtained. The purpose of this paper is to generalize Theorem II of Chudnovsky [3], in both the archimedian and the $p$-adic case. Our proof

(C) 1989 Australian Mathematical Society 0263-6115/89 \$A2.00+0.00 
follows closely the main lines of Väänänen ([12], [13]), but here we essentially use the ideas of Chudnovsky [3] in the construction of approximation forms and apply the local to global technique, as in Bombieri [2].

\section{Notations and results}

Let $K$ be an algebraic number field of degree $d$ over $\mathbf{Q}$, and let $O_{K}$ denote the domain of integers in $K$. For every place $v$ of $K$ we write $d_{v}=\left[K_{v}: \mathbf{Q}_{v}\right]$. If the finite place $v$ of $K$ lies over the prime number $p$, we write $v \mid p$, for infinite place $v$ of $K$ we write $v \mid \infty$. We normalize the absolute value ||$_{v}$ so that

(i) if $v \mid p$, then $|p|_{v}=p^{-d_{v} / d}$,

(ii) if $v \mid \infty$, then $|x|_{v}=|x|^{d_{v} / d}$, here || denotes the ordinary absolute value in $\mathbf{R}$ or $\mathbf{C}$.

The absolute height $h(x)$ of $x \in K$ is defined by the formula

$$
h(x)=\prod_{v} \max \left(1,|x|_{v}\right) .
$$

For any polynomial $P(z)=\sum_{i=0}^{n} p_{i} z^{i} \in K[z]$ we denote

$$
|P|_{v}=\max \left(1, \max _{i}\left|p_{i}\right|_{v}\right),
$$

and define the absolute height of $P$ by $h(P)=\prod_{v}|p|_{v}$.

We write $\log a=\log \max (1, a)$ for all $a \geq 0$, and denote

$$
\alpha_{v}=\left\{\begin{array}{ll}
1, & \text { if } v \mid p, \\
0, & \text { if } v \mid \infty,
\end{array} \quad \beta_{v}= \begin{cases}0, & \text { if } v \mid p, \\
d_{v} / d, & \text { if } v \mid \infty\end{cases}\right.
$$

The power series

$$
y_{i}(z)=\sum_{m=0}^{\infty} a_{m, i} z^{m}, \quad i=1, \ldots, n,
$$

are said to belong to the class $K G\left(\gamma, C, C_{0}\right), \gamma, C, C_{0} \geq 1$, if the following conditions are satisfied:

(i) $a_{m, i} \in K, i=1, \ldots, n, m=0,1, \ldots$;

(ii) $\max _{i}\left|a_{m, i}\right|_{v} \leq \gamma^{\beta_{v}} C^{\beta_{v} m}, m=0,1, \ldots$, for every $v \mid \infty$;

(iii) there exists a sequence of natural numbers $\left(r_{l}\right)$ such that

$$
r_{l} a_{m, i} \in O_{K}, \quad i=1, \ldots, n, \quad m=0,1, \ldots, l, \quad l=0,1, \ldots,
$$

and

$$
r_{l} \leq \gamma C_{0}^{l}, \quad l=0,1, \ldots
$$


By (iii),

$$
\max _{\substack{1 \leq i \leq n \\ 0 \leq m \leq l}}\left|a_{m, i}\right|_{v} \leq 1 /\left|r_{l}\right|_{v} \leq r_{l} \leq \gamma C_{0}^{l}
$$

for every finite place $v$ of $K$ and $l=0,1, \ldots$ Thus the functions (1) are $v$-adically convergent in

$$
|z|_{v}<C^{-\beta_{v}} C_{0}^{-\alpha_{v}} \text {. }
$$

In the following we suppose that the functions (1) satisfy a system of linear differential equations

$$
\frac{d}{d z} Y=A Y,
$$

where $Y=\left(y_{1}(z), \ldots, y_{n}(z)\right)^{\ell}, A=\left(A_{i j}(z)\right)_{n \times n}, A_{i j} \in K(z)$. Let $T(z) \in K[z]$ denote the common denominator of $A_{i j}$, and put

$$
s=\max \left(\operatorname{deg} T, \operatorname{deg} T A_{i j}, i, j=1, \ldots, n\right) .
$$

In the following theorem we shall estimate the $v$-value of

$$
P\left(y_{1}(\theta), \ldots, y_{n}(\theta)\right),
$$

$\theta \in K, P \in K\left[x_{1}, \ldots, x_{n}\right]$. In writing $\mid P\left(y_{1}(\theta), \ldots,\left.y_{n}(\theta)\right|_{v}\right.$ we consider all the coefficients of $P$ and the power series $y_{i}$ as elements of the corresponding completion $K_{v}$, and thus this $v$-value is defined for all $|\theta|_{v}<C^{-\beta_{v}} C_{0}^{-\alpha_{v}}$.

THEOREM. Assume that the functions (1) satisfying (2) are algebraically independent over $K(z)$ and belong to the class $K G\left(\gamma, C, C_{0}\right)$. Let

$$
P \in K\left[x_{1}, \ldots, x_{n}\right], \quad P \neq 0,
$$

be a polynomial of degree at most $\lambda$ and height $h(P)$. There then exist positive constants $c, \Lambda$, depending only on the functions (1) and $n$, such that, for any $\theta \in K$ of height $h(\theta) \leq h \geq e^{e}$ satisfying

$$
\begin{gathered}
\theta T(\theta) \neq 0, \quad \log h \geq(1+\max (3, \lambda))^{4 n} \log \log h, \\
|\theta|_{v}<e^{-c \lambda(\log h)^{(4 n-1) / 4 n}(\log \log h)^{1 / 4 n}}
\end{gathered}
$$

we have

$$
\left|P\left(y_{1}(\theta), \ldots, y_{n}(\theta)\right)\right|_{v}>h(P)^{-\Lambda(\log h)^{1 / 4}(\log \log h)^{-1 / 4}}
$$

for all $h(P) \geq H$, where

$$
\log H=\max \left\{c \lambda(\log h)^{2-1 / 4 n}(\log \log h)^{(1-4 n) / 4 n}, \log \max _{i}\left(1,\left|y_{i}(\theta)\right|_{v}\right)\right\}
$$

with a constant $\bar{c}>0$ depending only on (2).

We note that our condition (3) slightly sharpens the corresponding condition of Chudnovsky [3]. In fact, we shall prove the above estimate for $|P(\theta)|_{v}$ 
under a condition (3)' (see Section 4), which is better that (3) in some cases. Further, it should be noted that $4 n$ in (3) can be replaced by $2 n$ under the restrictive conditions used in Bombieri [2] or Väänänen [12], and then also $1 / 4$ in the conclusion can be replaced by $1 / 2$.

The authors thank the referee for his useful suggestions and advise. In particular, a bound for $H$ was not given in the original manuscript.

\section{Lemmas}

Let

$$
g_{0}(z) \equiv 1, \quad g_{1}(z), \ldots, g_{m}(z)
$$

denote the power-products

$$
y_{1}^{k_{1}}(z) \cdots y_{n}^{k_{n}}(z), \quad 0 \leq k_{1}+\cdots+k_{n} \leq S, \quad m=\left(\begin{array}{c}
n+S \\
S
\end{array}\right)-1,
$$

where $S$ is a natural number $\geq 3$. As in [12], we see that the functions (4) belong to the class $K G\left((2 \gamma)^{S}, 2 C, C_{0}^{1+(\log S)^{u}}\right)$ for some $u$ satisfying $0 \leq u \leq 1$. Thus these functions are $v$-adically defined in $|z|_{v}<(2 C)^{-\beta_{v}} C_{0}^{-\alpha_{v}\left(1+(\log S)^{u}\right)}$. Further, the functions (4) satisfy a system of linear differential equations of type (2), where the rational function coefficients again have a common denominator $T(z)$.

First we give a lemma on Padé approximations of the second kind (for the definition, see Chudnovsky [3]).

LEMMA 1. For any $\delta, 0<\delta<1 / m$, and an arbitrary positive integer $D$ and $M=\left[\left(m^{-1}-\delta\right) D\right]$, there exists a system $\left(Q(x) ; P_{1}(z), \ldots, P_{m}(z)\right)$ of Padé approximations of the second kind with parameters $(D, D, M)$ for the functions (4) such that $Q(z), P_{i}(z) \in K[z]$ and

$$
\begin{aligned}
\log h(Q) \leq & \left((\delta m)^{-1}-1\right)\left(1+m^{-1}-\delta\right) D\left(\log 2 C+\left(1+(\log S)^{u}\right) \log C_{0}\right) \\
& +(\delta m)^{-1}(\log 2(D+1)+2 S \log 2 \gamma+\log \Gamma),
\end{aligned}
$$

where $\Gamma$ is a positive constant depending only on $K$.

Proof. The proof is completely analogous to that of Lemma 4 in Väänänen and $\mathrm{Xu}$ [14], using Siegel's lemma in the form given by Bombieri [2].

The following result is Theorem 1.1 of Chudnovsky [3]. 
Lemma 2. Let $\left(Q(z) ; P_{1}(z), \ldots, P_{n}(z)\right)$ be a system constructed in Lemma 1. Let $k \in N$ and suppose that $M \geq k(s+1)$. We define

$$
\begin{aligned}
& Q^{\langle k\rangle}(z)=T^{k}(z)\left(\frac{d}{d z}\right)^{k} Q(z) / k !, \\
& \left.P_{i}^{\langle k\rangle}(z)=Q^{\langle k\rangle}(z) g_{i}(z)\right]_{D+k s}, \quad i=1, \ldots, m
\end{aligned}
$$

(this means that $P_{i}^{\langle k\rangle}(z)$ is a polynomial of degree $\leq D+k s$ such that the order of zero of $Q^{\langle k\rangle}(z) g_{i}(z)-P_{i}^{\langle k\rangle}(z)$ at $z=0$ is at least $\left.D+k s+1\right)$. Then $\left(Q^{\langle k\rangle}(z)\right.$; $\left.P_{1}^{\langle k\rangle}(z), \ldots, P_{m}^{\langle k\rangle}(z)\right)$ is a system of Padé approximations of the second kind with parameters $(D+k s, D+k s, M-k(s+1))$ for the functions (4).

Analogously to Lemma 5 of Väänänen and $\mathrm{Xu}$ [14], we now have the following

Lemma 3. Let $\left(Q(z) ; P_{1}(z), \ldots, P_{m}(z)\right)$ be a system constructed in Lemma 1. Let $k \in N$ and assume that $M \geq k(s+1)$. Then the system $\left(Q^{\langle k\rangle}(z)\right.$; $\left.P_{1}^{\langle k\rangle}(z), \ldots, P_{m}^{\langle k\rangle}(z)\right)$ defined in Lemma 2 has the following properties:

$\left|r_{D+k s} Q^{\langle k\rangle}\right|_{v} \leq\left(C(k, D)(2 \gamma)^{S} C_{0}^{\left(1+(\log S)^{u}\right)(D+k s)}\right)^{\beta_{v}}|Q|_{v}|T|_{v}^{k}$

$\left|r_{D+k s} P_{i}^{\langle k\rangle}\right|_{v} \leq\left.\left.\left(C(k, D)^{2}(2 \gamma)^{2 S}\left(2 C C_{0}^{1+(\log S)^{u}}\right)^{D+k s}\right)^{\beta_{v}}|Q|_{v}\right|_{T}\right|_{v} ^{k}, \quad i=1, \ldots, m$, where $C(k, D)=(k+1)(s+1)^{k}(D+1) 2^{D}$.

Let us denote, for all $k=0,1, \ldots, Q_{0}^{\langle k\rangle}=r_{D+k s} Q^{\langle k\rangle}, Q_{i}^{\langle k\rangle}=r_{D+k s} P_{i}^{\langle k\rangle}$, $i=1, \ldots, m$. We then have the following lemma, analogous to Lemma 2 of Väänänen and $\mathrm{Xu}[14]$.

LEMMA 4. Let $\delta, 0<\delta<1 /\left(m+m^{2}(s+1)\right)$, be given, and let $\theta \in K$ satisfy $\theta T(\theta) \neq 0$. There exists a positive constant $c_{0}$, depending only on the system (2), such that, for all

$$
D>N=c_{0}\left(m^{-1}-\delta\right)^{-1} S m^{2},
$$

there exist integers $k_{0}, k_{1}, \ldots, k_{m}$,

$$
0 \leq k_{0}<k_{1}<\cdots<k_{m} \leq J=D-m M+m(m+1)(s+1) / 2,
$$

satisfying

$$
\Delta(\theta)=\left|\begin{array}{cccc}
Q_{0}^{\left\langle k_{0}\right\rangle}(\theta) & Q_{1}^{\left\langle k_{0}\right\rangle}(\theta) & \ldots & Q_{m}^{\left\langle k_{0}\right\rangle}(\theta) \\
\vdots & \vdots & & \vdots \\
Q_{0}^{\left\langle k_{m}\right\rangle}(\theta) & Q_{1}^{\left\langle k_{m}\right\rangle}(\theta) & \ldots & Q_{m}^{\left\langle k_{m}\right\rangle}(\theta)
\end{array}\right|
$$


Proof. We prove here that the determinant

$$
\nabla(x)=\left|\begin{array}{ccc}
Q^{\langle 0\rangle}(x) & \ldots & Q^{\langle m\rangle}(x) \\
P_{1}^{\langle 0\rangle}(x) & \ldots & P_{1}^{\langle m\rangle}(x) \\
. & \ldots & . \\
P_{m}^{\langle 0\rangle}(x) & \ldots & P_{m}^{\langle m\rangle}(x)
\end{array}\right|
$$

is not identically zero for $D>N$. Then the proof follows immediately from the important Theorem 1.2 of Chudnovsky [3]. In our proof we follow Chudnovsky [3], Section 3.

Suppose the $\nabla(x) \equiv 0$. Let $1 \leq m$ be the integer such that the first $l$ columns of $\nabla$ are linearly independent over $\mathbb{C}(x)$, but the $(l+1)$ st column is linearly dependent on them. Let $F$ denote the matrix formed by these $l$ columns, and let $R$ and $U$ denote the matrices formed by the first $l$ rows and last $m-l+1$ rows of $F$, respectively. We may assume, without loss of generality, that det $R \not \equiv 0$.

Following Nesterenko [7], Section 3, we see that rational functions elements of the matrix

$$
U R^{-1}=\left(e_{i j}(x) / e(x)\right), \quad e_{i j}, e \in \mathbb{C}(x),
$$

satisfy $\max \left(\operatorname{deg} e_{i j}, \operatorname{deg} e\right) \leq c(1) S m$, where the constant $c(1)>0$ depends only on the system (2). Denote by $G$ the $l \times(m+1)$ matrix with $l$ rows $\left(g_{i}(x), 0, \ldots,-\delta_{i+1, j}, \ldots, 0\right), i=1, \ldots, l$, and let $G_{0}$ and $G_{1}$ denote the matrices formed by the first $l$ column and the last $m-l+1$ last columns of $G$, respectively. Denoting $T=G F$ we see, as in [3], Section 3, that

$$
\operatorname{ord}_{x=0} \operatorname{det}\left(e(x) T R^{-1}\right) \geq l(M-(l-1)(s+1)) .
$$

On the other hand,

$$
e(x) T R^{-1}=e(x) G_{0}+G_{1} E,
$$

where $E=\left(e_{i j}(x)\right)$. Thus $\operatorname{det}\left(e(x) T R^{-1}\right)$ is a polynomial in $x, y_{1}(x), \ldots$, $y_{n}(x)$, say $P\left(x, y_{1}(x), \ldots, y_{n}(x)\right)$, satisfying $\operatorname{deg}_{x} P \leq c(1) S m l, \operatorname{deg}_{y} P \leq S$. By the algebraic independence of $y_{1}, \ldots, y_{n}$ we know that $P$ is not identically zero in $x$. Using the result of Bertrand and Beukers [1] we obtain the estimate

$$
\operatorname{ord}_{x=0} \operatorname{det}\left(e(x) T R^{-1}\right) \leq c(1) S / m^{2}+c(2) m^{2}
$$

with positive constant $c(2)$ depending only on (2). Thus

$$
l(M-(l-1)(s+1)) \leq c(1) S l m^{2}+c(2) m^{2} .
$$

The above inequality is impossible for all $\left(m^{-1}-\delta\right) D>c_{0} S m^{2}$ with some positive constant $c_{0}$ depending only on (2). This proves our Lemma 4 .

We next define rational functions $L_{t, j}=L_{t, j}(\theta), t, j=0,1, \ldots, m$, as the solutions of the system

$$
\sum_{t=0}^{m} L_{t, j} Q_{t}^{\left\langle k_{i}\right\rangle}(\theta)=\delta_{i, j}, \quad i, j=0,1, \ldots, m,
$$


of linear equations. By Cramer's rule,

$$
L_{t, j}(\theta)=R_{t, j}(\theta) / \Delta(\theta), t, \quad j=0,1, \ldots, m,
$$

where $R_{t, j}(\theta)$ is the $t, j$-cofactor of the matrix corresponding to $\Delta(\theta)$. We now define linear forms $F_{j}$ in $g_{0}(\theta), g_{1}(\theta), \ldots, g_{m}(\theta)$ by the formulae

$$
F_{j}(\theta)=\sum_{t=0}^{m} M_{t, j}(\theta) g_{t}(\theta), \quad j=0,1, \ldots, m,
$$

where $M_{t, j}(\theta)=R_{t, j}(\theta) \theta^{-\omega}, \omega=(m-1)(M+D)=(m-1) J$. Using Theorem 4.1 of Chudnovsky [3] we immediately obtain the following important result.

LEMMA 5. Let the hypothesis of Lemma 4 be satisfied. For all $D>N$, the linear forms $F_{0}(\theta), \ldots, F_{m}(\theta)$ in $g_{0}(\theta), \ldots, g_{m}(\theta)$ are linearly independent and have polynomial coefficients $M_{t, j}=M_{t, j}(\theta)$ satisfying

$$
\operatorname{deg}_{\theta} M_{l, j} \leq D-(m-1) M+J(m s+m-1), \quad t, j=0,1, \ldots, m .
$$

Further, we have

$$
\operatorname{ord}_{\theta=0} F_{j}(\theta) \geq D+M-J, \quad j=0,1, \ldots, m .
$$

LEMMA 6. The polynomials $M_{t, j}$ appearing in Lemma 5 satisfy the estimate

$$
\begin{aligned}
&\left|M_{t, j}\right|_{v} \leq(m !)^{\beta_{v}}\left((D+J s+1) C(J, D)^{2}(2 \gamma)^{2 S}\right. \\
&\left.\cdot\left(2 C C_{0}^{\left(1+(\log S)^{\mu}\right)}\right)^{(D+J s)}\right)^{m \beta_{v}}|Q|_{v}^{m}|T|_{v}^{m J}, \\
& t, j=0,1, \ldots, m .
\end{aligned}
$$

Proof. The result follows immediately from Lemma 3 and the definition of the polynomials $M_{t, j}$.

Lemma 7. Let $\delta, 0<\delta<1 /\left(3 m^{2}(s+1)\right)$, be given. Assume that

$$
D>\max \left\{\delta^{-1}(1+(m+1)(s+1) / 2), m /\left(1-3 \delta m^{2}(s+1)\right), N\right\} .
$$

If $\theta \in K$, then

$$
\begin{aligned}
\left|M_{t, j}(\theta)\right|_{v} \leq & \left(D\left(m^{-1}+\delta\left(m+2 m^{2}(s+1)\right)\right)+1\right)^{\beta_{v}}\left|M_{t, j}\right|_{v} \\
& \cdot \max \left(1,|\theta|_{v}^{D\left(m^{-1}+\delta\left(m+2 m^{2}(s+1)\right)\right)}\right), \quad t, j=0,1, \ldots, m .
\end{aligned}
$$

Further, if $|\theta|_{v}<(4 C)^{-\beta_{v}} C_{0}^{-\alpha_{v}\left(1+(\log S)^{u}\right)}$, then we have the estimates

$$
\begin{array}{r}
\left|F_{j}(\theta)\right|_{v} \leq(2 \gamma)^{S\left(\beta_{v}+\alpha_{v}\right)}\left(2(m+1)\left(D\left(m^{-1}+\delta\left(m+2 m^{2}(s+1)\right)\right)+1\right)^{\beta_{v}}\right. \\
\cdot \max _{t, j}\left|M_{t, j}\right|_{v}\left((2 C)^{\beta_{v}} C_{0}^{\alpha_{v}\left(1+(\log S)^{u}\right)}|\theta|_{v}\right)^{D\left(1+m^{-1}-3 \delta m\right)}, \\
j=0,1, \ldots, m .
\end{array}
$$


Proof. It follows from the hypothesis that the hypotheses of Lemmas 4, 5 and 6 are valid, and $J \leq 2 \delta m D$. In addition, we obviously have

$$
D-(m-1) M+J(m s+m-1) \leq D\left(m^{-1}+\delta\left(m+2 m^{2}(s+1)\right)\right)
$$

and

$$
D+M-J \geq\left(1+m^{-1}-3 \delta m\right) D .
$$

The estimate for $\left|M_{t, j}(\theta)\right|_{v}$ now follows from Lemma 5 . To prove the second estimate, we write

$$
M_{t, j}(\theta)=\sum_{l=0}^{R} m_{t, j, l} \theta^{l}, \quad g_{t}(\theta)=\sum_{i=0}^{\infty} g_{t, i} \theta^{i},
$$

where $R \leq D\left(m^{-1}+\delta\left(m+2 m^{2}(s+1)\right)\right)$, by Lemma 5 . Lemma 5 also implies

$$
\begin{aligned}
F_{j}(\theta) & =\sum_{t=0}^{m} M_{t, j}(\theta) g_{t}(\theta)=\sum_{i \geq D+M-J}\left(\sum_{t=0}^{m} \sum_{l=0}^{\min (i, R)} m_{t, j, l} g_{t, i-l}\right) \theta^{i} . \\
\mid F_{j}(\theta)_{v} & \leq \sum_{i \geq D+M-J}\left|\sum_{t=0}^{m} \sum_{l=0}^{\min (i, R)} m_{t, j, l} g_{t, i-l}\right|_{v}|\theta|_{v}^{i} \\
& \leq((m+1)(R+1))^{\beta_{v}} \max _{t, j}\left|M_{t, j}\right|_{v}(2 \gamma)^{S \beta_{v}} \sum_{i \geq D+M-J}\left((2 C)^{\beta_{v}}|\theta|_{v}\right)^{i} \\
& \leq\left(2^{S+1} \gamma^{S}(m+1)(R+1)\right)^{\beta_{v}} \max _{t, j}\left|M_{t, j}\right|_{v}\left((2 C)^{\beta_{v}}|\theta|_{v}\right)^{\left(1+m^{-1}-3 \delta m\right) D} .
\end{aligned}
$$

In the case $v \mid p$ we have $\left|r_{i} g_{t, i-l}\right| v \leq 1$, which implies

$$
\left|g_{t, i-l}\right|_{v} \leq 1 /\left|r_{i}\right|_{v} \leq r_{i} \leq(2 \gamma)^{S} C_{0}^{\left(1+(\log S)^{u}\right) i}
$$

Thus

$$
\left|F_{j}(\theta)\right|_{v} \leq \max _{t, j}\left|M_{t, j}\right|_{v}(2 \gamma)^{S}\left(C_{0}^{1+(\log S)^{u}}|\theta|_{v}\right)^{\left(1+m^{-1}-3 \delta m\right) D} .
$$

This proves Lemma 7.

\section{Proof of the Theorem}

The main lines of the proof follow the work Väänänen [12]. Let $\theta \in K$ satisfy $h(\theta) \leq h \geq e^{e}$ and

$$
\left\{\begin{array}{l}
\theta T(\theta) \neq 0, \quad \log h \geq(1+\max (3, \lambda))^{4 n}(\log \log h)^{u}, \\
|\theta|_{v}<e^{-c \lambda(\log h)^{(4 n-1) / 4 n}(\log \log h)^{u / 4 n}},
\end{array}\right.
$$


where $c$ will be given in (6). We define the natural number $S$ by $S=\left[l(h)^{1 / 4 n}\right]$, where $l(h)=(\log h) /(\log \log h)^{u}$, and denote

$$
t=\left(\begin{array}{c}
n+S-\lambda \\
n
\end{array}\right), \quad w=m+1=t .
$$

As in Väänänen [12], we obtain the estimates

$$
S^{n} / n ! \leq m \leq c_{1} S^{n}, \quad t<m, w \leq c_{2} \lambda S^{n-1},
$$

with positive constants $c_{1}$ and $c_{2}$ depending only on $n$. For the constant $c$ appearing in (3) or (3)' we take the value

$$
c=c_{1}^{3} c_{2} A /(4 n)^{u}+3 c_{2}(n !)+1,
$$

where

$$
A=4(s+3)\left(\log 4 \gamma \Gamma C C_{0}^{2}+1\right)+\log h(T) .
$$

We now choose a natural number $D$ in such a way that

$$
\begin{gathered}
(D-1) \lambda(\log h)^{(4 n-1) / 4 n}(\log \log h)^{u / 4 n} \leq t \log h(P) \\
<D \lambda(\log h)^{(4 n-1) / 4 n}(\log \log h)^{u / 4 n} .
\end{gathered}
$$

Here we assume $h(P)$ to be large enough, say $h(P) \geq H_{0}$, that $D$ satisfies the conditions of Lemma 7:

$$
D>\max \left\{\delta^{-1}(1+(m+1)(s+1) / 2), m /\left(1-3 \delta m^{2}(s+1)\right), N\right\},
$$

where we choose

$$
\delta=1 /\left(2 m\left(m+2 m^{2}(s+1)\right)\right) .
$$

By the definitions of $D$ and $N$ it follows that we may choose

$$
\log H_{0}=\tau \lambda(\log h)^{2-1 / 4 n}(\log \log h)^{u(1-4 n) / 4 n},
$$

where $c>0$ is a constant depending only on (2).

By multiplying the polynomial $P\left(y_{1}(z), \ldots, y_{n}(z)\right)$ by the power-products

$$
y_{1}^{k_{1}}(z) \cdots y_{n}^{k_{n}}(z), 0 \leq k_{1}+\cdots+k_{n} \leq S-\lambda,
$$

we obtain linear forms in $g_{0}(z), \ldots, g_{m}(z)$, say

$$
\psi_{i}(z)=\sum_{j=0}^{m} a_{j, i} g_{j}(z), \quad i=1, \ldots, t,
$$

where the $a_{j, i}$ are the coefficients of $P$ or zero.

We now use Lemma 5 to find $w$ linear forms, say $F_{j_{k}}(\theta), k=1, \ldots, w$, such that these forms together with the forms $\psi_{i}(\theta), i=1, \ldots, t$, are linearly independent (by $(3)^{\prime}$, we have $\left.|\theta|_{v}<(4 C)^{-\beta_{v}} C_{0}^{-\alpha_{v}\left(1+(\log S)^{\mu}\right)}\right)$. Then the 
determinant of these forms, say

$$
\Delta_{1}(\theta)=\left|\begin{array}{ccc}
a_{01} & \ldots & a_{m 1} \\
\vdots & & \vdots \\
a_{0 t} & \ldots & a_{m t} \\
M_{0, j_{1}}(\theta) & \ldots & M_{m, j_{1}}(\theta) \\
\vdots & & \vdots \\
M_{0, j_{w}}(\theta) & \ldots & M_{m, j_{w}}(\theta)
\end{array}\right|
$$

must differ from zero. This determinant $\Delta_{1}(\theta) \in K$, and thus the product formula gives $\Pi_{v}\left|\Delta_{1}(\theta)\right|_{v}=1$.

By the above formula, we first obtain a lower bound

$$
\begin{aligned}
\log \left|\Delta_{1}(\theta)\right|_{v} & =-\sum_{v_{1} \neq v} \log \left|\Delta_{1}(\theta)\right|_{v_{1}} \\
\geq & -\sum_{v_{1} \neq v}\left(\beta_{v_{1}}(m+1) \log (m+1)+t \log |P|_{v_{1}}\right. \\
& \left.+w \log \max _{i, k}\left|M_{i, j_{k}}(\theta)\right|_{v_{1}}\right) .
\end{aligned}
$$

On the other hand,

(8) $\left|\Delta_{1}(\theta)\right|_{v} \leq(m+1)^{\beta_{v}} \max \left\{\max _{1 \leq j \leq t}|\operatorname{cofactor}(1, j)|_{v}\left|\Psi_{j}(\theta)\right|_{v}\right.$,

$$
\text { - } \left.\max _{1 \leq i \leq w}|\operatorname{cofactor}(1, t+\dot{i})|_{v}\left|F_{j_{i}}(\theta)\right|_{v}\right\} \text {, }
$$

and here cofactor $(1, j)$ means the $1, j$-cofactor of the matrix corresponding to $\Delta_{\mathrm{l}}(\theta)$.

Since

$$
\begin{aligned}
\log \left((m+1)^{\beta_{v}}\right. & \left.\max _{1 \leq i \leq w} \mid \text { cofactor }\left.(1, t+i)\right|_{v}\left|F_{j_{i}}(\theta)\right|_{v}\right) \\
\leq & \beta_{v}(m+1) \log (m+1)+t \log |P|_{v} \\
& +(w-1) \log \max _{i, k}\left|M_{i, j_{k}}(\theta)\right|_{v}+\log \max _{i}\left|F_{j_{i}}(\theta)\right|_{v},
\end{aligned}
$$

we have, by Lemma 7, the upper estimate

(9) $\log \left((m+1)^{\beta_{v}} \max _{1 \leq i \leq w}|\operatorname{cofactor}(1, t+i)|_{v}\left|F_{j_{i}}(\theta)\right|_{v}\right)-\log \left|\Delta_{1}(\theta)\right|_{v}$

$$
\begin{aligned}
& \leq(m+1) \log (m+1)+t \log h(P) \\
& +w \sum_{v_{1} \neq v} \log ^{+} \max _{i, k}\left|M_{i, j_{k}}(\theta)\right|_{v_{1}}+(w-1) \log ^{+} \max _{i, k}\left|M_{i, j_{k}}(\theta)\right|_{v} \\
& +\log \left\{(2 \gamma)^{S\left(\beta_{v}+\alpha_{v}\right)}\left(2\left(m_{1}\right) D\left(m^{-1}+\delta\left(m+2 m^{2}(s+1)\right)\right)+1\right)^{\beta_{v}}\right. \\
& \left.\cdot \max _{t, j}\left|M_{t, j}\right|_{v}\left((2 C)^{\beta_{v}} C_{0}^{\alpha_{v}\left(1+(\log S)^{u}\right)}|\theta|_{v}\right)^{D\left(1+m^{-1}-3 \delta m\right)}\right\} .
\end{aligned}
$$


Using Lemma 1, 6 and 7 we see that (9) is smaller than

$$
\begin{aligned}
&(m+1) \log (m+1)+t \log h(P) \\
& \quad+w \sum_{v_{1}}\left\{\beta_{v_{1}} \log (2 D / m)+D\left(m^{-1}+\delta\left(m+2 m^{2}(s+1)\right)\right) \log ^{+}|\theta|_{v_{1}}\right. \\
& \quad+\beta_{v_{1}} m \log m+m \beta_{v_{1}}(\log (D+J s+1)+2 \log C(J, D) \\
& \quad+2 S \log 2 \gamma+(D+J s)\left(\log 2 C+\left(1+(\log S)^{u}\right) \log C_{0}\right) \\
&\left.\quad+m \log |Q|_{v_{1}}+m J \log |T|_{v_{1}}\right\} \\
& \quad \\
& \quad+S\left(\beta_{v}+\alpha_{v}\right) \log 2 \gamma+\beta_{v} \log 2(m+1) \quad \\
& \quad+D\left(1+m^{-1}-3 \delta m\right)\left(\beta_{v} \log 2 C+\alpha_{v}\left(1+(\log S)^{u}\right) \log C_{0}\right) \\
& \quad+D \log |\theta|_{v} \leq w m D(\log h(T)+2 \log 2 \gamma+10+3 \log 2 C) \\
& \quad+3 w m D\left(1+(\log S)^{u}\right) \log C_{0}+w m \log h(Q) \\
& \quad+2 w m^{-1} D \log h(\theta)+t \log h(P)+D \log |\theta|_{v} \\
& \leq A w m^{3} D(\log S)^{u}+2 w m^{-1} D \log h(\theta)+t \log h(P)+D \log |\theta|_{v} \\
& \leq A c_{1}^{3} c_{2} \lambda S^{4 n-1}(\log S)^{u} D+2 c_{2}(n !) \lambda S^{-1} D \log h(\theta)+t \log h(P)+D \log |\theta|_{v} \\
& \leq(c-1) \lambda(\log h)^{(4 n-1) / 4 n}(\log \log h)^{u / 4 n} D+t \log h(P)+D \log |\theta|_{v} \\
& \leq t \log h(P)-D \lambda(\log h)^{(4 n-1) / 4 n}(\log \log h)^{u / 4 n}<0 .
\end{aligned}
$$

It thus follows from (7) and (8) that

$$
\begin{aligned}
& -\sum_{v_{1} \neq v}\left(\beta_{v_{1}}(m+1) \log (m+1)+t \log |P|_{v+1}+w \log \max _{i, k}\left|M_{i, j_{k}}(\theta)\right|_{v_{1}}\right) \\
& \leq \log \left((m+1)^{\beta_{v}} \max _{1 \leq j \leq l}|\operatorname{cofactor}(1, j)|_{v}\left|\Psi_{j}(\theta)\right|_{v}\right) .
\end{aligned}
$$

Completely analogously to the above deduction we now obtain

$$
\begin{gathered}
\log \max _{1 \leq j \leq t}\left|\Psi_{j}(\theta)\right|_{v}>-\left(t \log h(P)+(c-1) \lambda(\log h)^{(4 n-1) / 4 n}(\log \log h)^{u / 4 n} D\right) \\
>-(c+1) t \log h(P)>-(c+1) c_{1} l(h)^{1 / 4} \log h(P) .
\end{gathered}
$$

Since

$$
\max _{1 \leq j \leq t}\left|\Psi_{j}(\theta)\right|_{v} \leq \max _{i}\left(1,\left|y_{i}(\theta)\right|_{v}\right)^{S}\left|P\left(y_{1}(\theta), \ldots, y_{n}(\theta)\right)\right|_{v},
$$

the truth of the Theorem follows.

\section{Acknowledgement}

The second author wishes to thank the Department of Mathematics of the University of Oulu for their support and hospitality. 


\section{References}

[1] D. Bertrand and F. Beukers, 'Équations différentielles linéaires et majorations de multiplicités,' Ann. Sci. École Norm. Sup. 18 (1985), 181-192.

[2] E. Bombieri, 'On $G$-functions,' Recent progress in analytic number theory, Vol. II, edited by H. Halberstam and C. Hooley (Academic Press, 1981).

[3] G. V. Chudnovsky, 'On applications of diophantine approximations,' Proc. Nat. Acad. Sci. U.S.A. 8 (1984), 7261-7265.

[4] Y. Flicker, 'On p-adic G-functions,' J. London Math. Soc. 15 (1977), 395-402.

[5] A. I. Galochkin, 'Lower bounds for polynomials of the values of the class of analytic functions,' Mat. Sb. 95 (137) (3) (1974), 396-417.

[6] E. M. Matveev, 'Linear forms in the values of $G$-functions, and diophantine equations,' Mat. Sb. 117 (159) (3) (1982), 379-396.

[7] Yu. V. Nesterenko, 'Estimates for the orders of zeros of functions of a certain class and applications in the theory of transcendental numbers,' Izv. Akad. Nauk SSSR Ser. Mat. 41 (1977), 239-270.

[8] M. S. Nurmagomedov, 'The arithmetic properties of the values of $G$-functions,' Vestnik Moskov. Univ. Ser. I, Mat. Meh. 26 (6) (1971), 79-86.

[9] C. L. Siegel, 'Uber einige Anwendungen diophantischer Approximationen,' Abh. Preuss. Akad. Wiss., Phys.-Mat. Kl. No. 1 (1929).

[10] K. Väänänen, 'On linear forms of a certain class of $G$-functions and $p$-adic $G$-functions,' Acta Arith. 36 (1980), 273-295.

[11] K. Väänänen, 'On the arithmetic properties of p-adic G-functions,' Arch. Math. 35 (1980), 364-373.

[12] K. Väänänen, 'On a class of $G$-functions,' Mathematics, University of Oulu 1/81, 1981.

[13] K. Väänänen, 'On a class of $G$-functions,' Progress in mathematics, 38, edited by J. Coates and S. Helgason (Birkhäuser, 1983).

[14] K. Väänänen and Xu Guangshan, 'On linear forms of G-functions,' Acta Arith. 50 (1988), 251-263.

[15] Xu. Guangshan, 'On lower estimate of linear forms involving a class of $G$-functions,' Acta Math. Sinica 24 (1981), 578-586.

[16] Xu. Guangshan, 'A note on linear fomrs in a class of $E$-functions and $G$-functions', $J$. Austral. Math. Soc. Ser. A 35 (1983), 338-348.

Department of Mathematics

University of Oulu

Institute of Mathematics

Oulu Academia Sinica

Finland

Beijing

China 